\title{
Accreting millisecond X-ray pulsars: recent developments
}

\author{
Rudy Wijnands \\ Astronomical Institute 'Anton Pannekoek', University of Amsterdam, Postbus 94249, 1090 GE \\ Amsterdam, the Netherlands \\ email: r.a.d.wijnands@uva.nl
}

\begin{abstract}
It is now more than eleven years since the discovery of the first accreting millisecond X-ray pulsar. Since then, eleven additional systems have been found, two of them during the last year. Here I briefly discuss the most recent developments with respect to these systems.
\end{abstract}

Keywords. pulsars:X-ray - stars:neutron - X-rays:binaries

\section{Introduction}

Since the discovery of the first accreting millisecond X-ray pulsar (AMXP) in 1998 (Wijnands \& van der Klis 1998), eleven additional systems have been found (see Table 1 and Section 2 for the two most recently discovered AMXPs). These systems are important because they are the evolutionary link between millisecond radio pulsars and accreting neutron stars in low-mass X-ray binariest. In addition, by studying the pulsations in the AMXPs, one hopes to learn about the physics of accretion onto a magnetized object and about the properties of neutron stars. Currently all AMXPs are transients and one must wait for the sources to go into outburst in order to study them and their pulsations. Here I present some highlights of the most recent developments related to the study of AMXPs.

\section{The two most recently discovered AMXPs}

In the months after the IAU General assembly, two new AMXPs were discovered. The source IGR J17511-3057 was discovered by INTEGRAL in early September 2009 (Baldovin et al. 2009) and soon afterwards it was found (using RXTE; Markwardt et al. 2009) to be an AMXP. The neutron star in this system has a spin frequency of $\sim 245 \mathrm{~Hz}$ and the orbital period of the system is $\sim 208$ minutes (Markwardt et al. 2009; see also Table 1). In addition burst oscillations were observed (Watts et al. 2009) and a near infra-red counterpart was discovered (Torres et al. 2009a,b). None of this makes this source exceptional; it would seem to be just a 'run-of-the-mill' AMXP. The second, recently discovered AMXP does exhibit some atypical behavior. Heinke et al. (2009) reported the discovery of a new X-ray transient in the gobular cluster NGC 6440 (the second one in this cluster). An RXTE observation on this cluster showed that this transient is an AMXP. The neutron star in this system has a spin period of $\sim 205 \mathrm{~Hz}$ and the orbital period is 57 min (Altamirano et al. 2009). These properties are not different from those of other known AMXPs. However, it was found (Heink et al. 2009) that this source has only very short outbursts (of order days) and the peak X-ray luminosity during outburst is only

$\dagger$ Recently, a different type of 'link' between millisecond radio pulsars and neutron-star lowmass X-ray binaries has been found (Archibald et al. 2009) opening up an additional, very interesting window leading to understanding the relationship between the two types of sources. 
Table 1. The currently known AMXPs with their basic characteristics and when the pulsations were first seen

\begin{tabular}{ccccc}
\hline Name & $\begin{array}{c}\text { spin }(\mathrm{Hz}) \\
(\mathrm{Hz})\end{array}$ & $\begin{array}{c}\text { binary period }(\mathrm{min}) \\
(\text { minutes })\end{array}$ & $\begin{array}{c}\mathrm{M}_{c, m i n}\left(M_{\odot}\right) \\
\text { When discovered }\end{array}$ & \\
\hline SAX J1808.4-3658 & 401 & 120 & 0.043 & April 1998 \\
XTE J1751-305 & 435 & 42 & 0.014 & April 2002 \\
XTE J0929-314 & 185 & 44 & 0.083 & April 2002 \\
XTE J1807-294 & 191 & 40 & 0.0066 & February 2003 \\
XTE J1814-338 & 314 & 258 & 0.17 & June 2003 \\
IGR J00291+5934 & 599 & 150 & 0.039 & December 2004 \\
HETE J1900.1-2455 & 377 & 84 & 0.016 & June 2005 \\
Swift J1756.9-2508 & 182 & 54 & 0.007 & June 2007 \\
Aql X-1 & 550 & 1140 & $?$ & August 2007 \\
SAX J1748.9-2021 & 442 & 526 & 0.1 & August 2007 \\
NGC 6440 X-2 & 206 & 57 & 0.0067 & August 2009 \\
IGR J17511-3057 & 245 & 208 & 0.0011 & September 2009 \\
\hline
\end{tabular}

$\sim 10^{36} \mathrm{erg} \mathrm{s}^{-1}$ which makes it rather difficult to study in outburst. However, soon after it was discovered it was found that the source appears to be in outbursts roughly every month. This makes it the X-ray transient with the shorted known recurrence time (Heinke et al. 2009). The reason for this short recurrence time is not understood. Currently, monitoring campaigns using RXTE and Swift have begun to observe more outbursts of this source in order to study the evolution of its pulsations over time. Its frequent outbursts might give us unique insight into magnetic accretion if enough high-time resolution data during outburst can be collected. The discovery of this source also raises the question if many similar systems are missed, especially if they have similar short and weak outbursts but which are less frequent.

With twelve AMXPs now known, the neutron-star spin distribution can be studied in low-mass X-ray binaries. By including also the neutron-star spin measurements obtained from nearly coherent oscillations during type-I X-ray bursts (the so-called burst oscillations) the sample of sources now reaches about thirty. It was noted with a smaller sample (e.g., Chakrabarty et al. 2003 and references to that paper) that the neutron-star spin distribution in low-mass X-ray binaries (and also in the millisecond radio pulsars) appears to cut-off at around 600 to $700 \mathrm{~Hz}$, with no neutron stars spinning faster than that $\nmid$ despite the fact that the break-up frequency of neutron stars is significantly larger. By using the full list of spin frequencies currently available, this conclusion still holds (see, e.g., Fig.1 which shows the sample complete until the summer of 2009 but does not include the most recent discoveries which are still consistent with this general picture). Currently, it is unclear what is the underlying physics. The most exciting explanation (although probably not the correct one) proposes that the spinning up of the neutron star in low-mass X-ray binaries is halted by the emission of gravitational waves from the stars (see Chakrabarty 2005 and Chakrabarty et al. 2003 and the papers referring to these two papers for a discussion of the various models).

$\dagger$ One system has a rather marginal indication of a burst-oscillation frequency (and thus a neutron-star spin) larger than $1000 \mathrm{~Hz}$, but this result is highly debated and needs to be confirmed. I will not discuss this system further. 


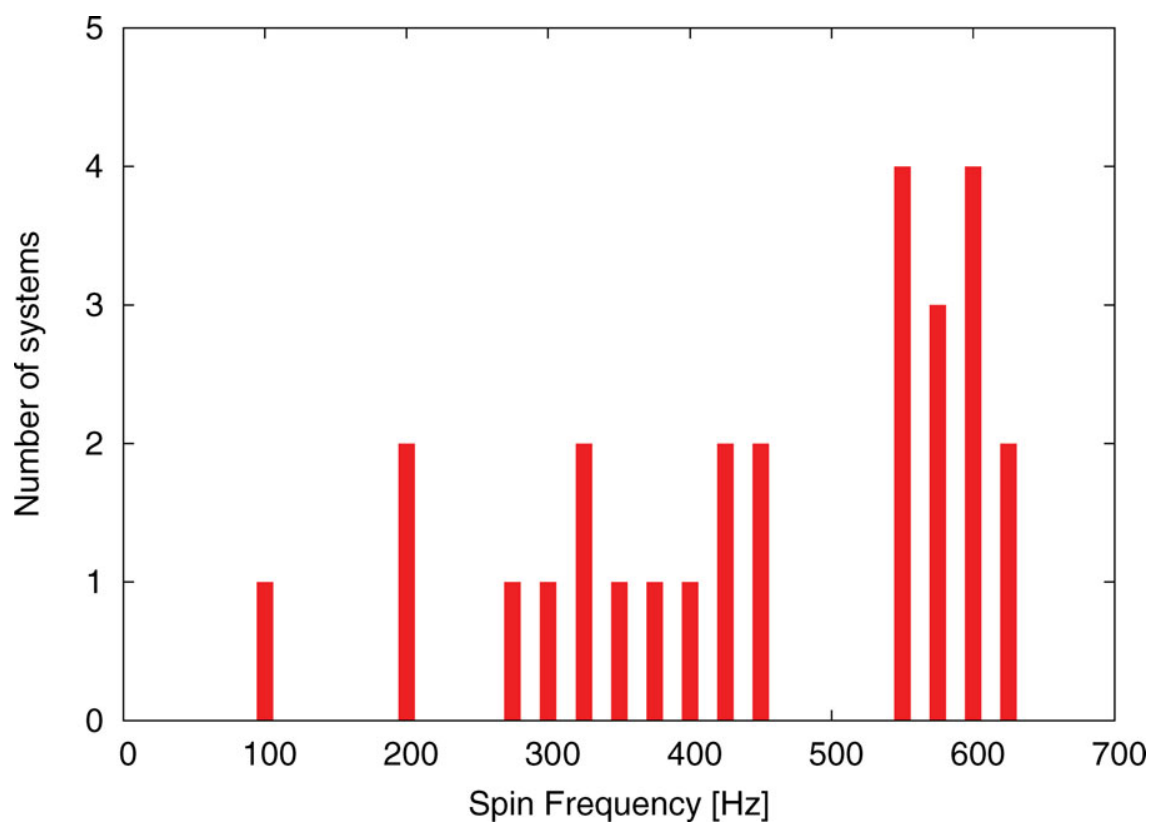

Figure 1. The number of AMXPs and burst oscillation sources (as of summer 2009) versus the observed frequency of the neutron stars (as inferred from the frequency of the pulsations or the burst oscillations). Figure made by Alessandro Patruno for the purpose of my presentation at the meeting.

\section{Timing noise}

One of the main reasons to study AXMPs is to determine the reaction of the neutron star to the accretion of matter. The usual way to investigate this is to search for changes in the pulse frequency (i.e., to determine a spin-frequency derivative or set upper limits on any). However, the measurement of such a frequency derivative has been severly hampered by an unmodeled component in the pulse arrival times which has been called 'timing noise' (named after a similar phenomenon, albeit on much longer time scales, seen in several radio pulsars). The presence of this timing noise has raised serious concerns about the validity of any frequency derivative reported in the literature. Recently, it has been shown that it is possible to attribute most of this timing noise to a phase offset of the pulsations that varies in correlation or anti-correlation with the X-ray flux (Patruno et al. 2009). If confirmed, this correlation could bias the measured spin frequencies and could artifically introduce a frequency derivative. Therefore it is very important to study all AMXPs (and hopefully also new ones) in detail to either confirm or reject this correlation. A confirmation would seriously inhibit our ability to measure any true spin period changes.. Interestingly, theoretically, these effects can rather easily be explained by assuming a wandering hot spot whose location depends on the accretion rate or by a systematic variations of the pulse profile (Patruno et al. 2009; Lamb et al. 2009, Poutanen et al. 2009).

\section{Why only so few accreting millisecond X-ray puslars}

Despite decades of observations, it is still unclear why only a dozen of the more than 150 known neutron-stars low-mass X-ray binaries exhibit pulsations. Aside from the pulsations, the other properties of the AMXPs look remarkably similar to those observed 


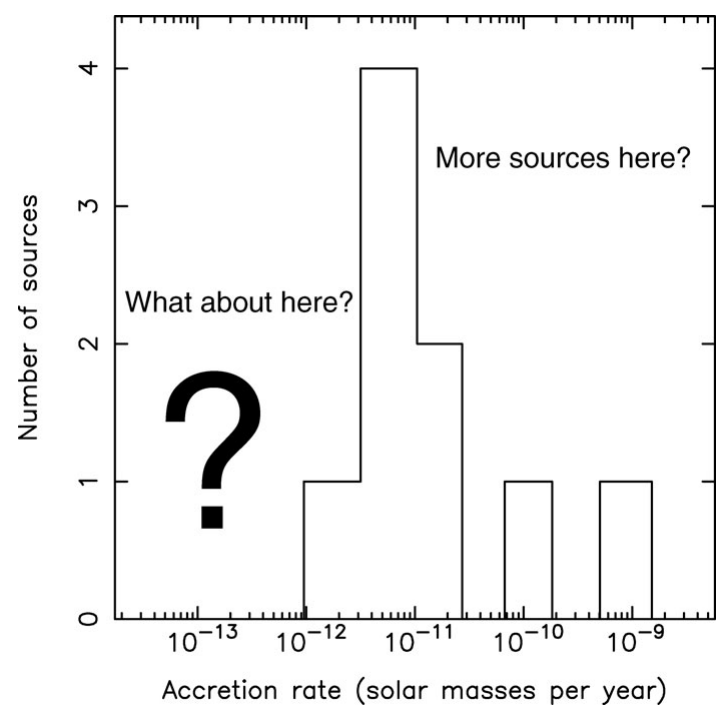

Figure 2. The number of AMXPs (as of summer 2009 and not including Aql X-1) versus their time averaged accretion rate.

from the non-pulsating sources, indicating that the difference between the two classess should be subtle. One possiblity is that the neutron stars in the non-pulsating systems have no or only a very weak magnetic field or that their magnetic axis is aligned with their spin axis. However, if this idea is correct, then those systems cannot be the progenitors of the millisecond radio pulsars unless a mechanism is available which would increase the magnetic field or misalign it with the rotation axis after the accretion has halted. Alternatively, it has been proposed that in the non-pulsating systems the pulsations (which are intrinsically there) are scatterred in a Comptonizing medium (e.g., Titarchuk et al. 2002) but it has been suggested that this cannot work for all systems (e.g., Gögüss et al. 2007; but see Titarchuk et al. 2007).

Based on the rather low time-averaged accretion rate $(\langle\dot{M}\rangle)$ of the first AMXP discovered (SAX J1808.4-3658), Cumming et al. (2001) suggested that if $\langle\dot{M}\rangle$ is sufficiently high, the neutron star magnetic field might be buried by the accreted matter and does not have time to dissipate through the accreted material. However, for SAX J1808.4-3658 $\langle\dot{M}\rangle$ is sufficiently low that the magnetic field dissipation can indeed happen, giving it a magnetic field still strong enough to disturb the flow of the accreted matter. Intriguinly, nearly all of the AMXPs discovered after this (see Figure 2) have indeed rather low $\langle\dot{M}\rangle$ which would support this idea. The only outlayers are the so-called intermittent AMXPs which do not always exhibit pulsations. Those systems have high time averaged accretion rates and the intermittency of their pulsations might be related to that. Still, things are not fully clear and the burying model does have problems explaining those systems (see the discussions in Galloway et al. 2007, Casella et al. 2008, and Altamirano et al. 2008).

In order to make progress in our understanding of the reason (or reasons) why only such a small percentage of the neutron-star low-mass X-ray binaries exhibit pulsations, more AMXPs need to be discovered. When looking at Figure 2 (adapted from Wijnands 2008a), two strategies can be envisaged to try to enlarge the sample of AMXPs. Firstly, one should investigate the brighter transients with typically high $\langle\dot{M}\rangle\left(>10^{-10} M_{\odot} \mathrm{yr}^{-1}\right)$ in detail for possible short lived episodes during which they migh exhibit pulsations. Some searches have been performed already using the RXTE archive but so far no new AMXP has been found. However, such searches still need to be fully optimized and several 
AMXPs might still be lurking in the RXTE archive. Secondly, if indeed most AMXPs have low $\langle\dot{M}\rangle$, one might assume (see Wijnands 2008a and references therein) that those neutron-star low-mass X-ray binaries with a very low $\langle\dot{M}\rangle\left(<10^{-11} M_{\odot} \mathrm{yr}^{-1}\right)$ might also exhibit coherent pulsations when in outburst. The fact that NGC 6440 X-2 might have a $\langle\dot{M}\rangle$ as low as $\sim 10^{-12} M_{\odot} \mathrm{yr}^{-1}$ (Heinke et al. 2009) would support that idea. However, those systems are difficult to find. RXTE might not be sensitive enough to detect the pulsations leaving only XMM-Newton to be used (see discussion in Wijnands 2008b). Therefore, the discovery of those systems and the search for possible pulsations is quite challenging but could yield very important results which would help us to understand why only so few systems pulsate.

\section{Acknowledgements}

I would like to thank Alessandro Patranski for the help in preparing Figure 1.

\section{References}

Archibald, A. M., et al. 2009, Science, 324, 1411

Altamirano, D., Casella, P., Patruno, A., Wijnands, R., \& van der Klis, M. 2008, ApJ, 674, L45

Altamirano, D., et al. 2009, arXiv:0911.0435

Baldovin, C., et al. 2009, The Astronomer's Telegram, 2196, 1

Casella, P., Altamirano, D., Patruno, A., Wijnands, R., \& van der Klis, M. 2008, ApJ, 674, L41

Chakrabarty, D. 2005, Binary Radio Pulsars, 328, 279

Chakrabarty, D., Morgan, E. H., Muno, M. P., Galloway, D. K., Wijnands, R., van der Klis, M., \& Markwardt, C. B. 2003, Nature, 424, 42

Cumming, A., Zweibel, E., \& Bildsten, L. 2001, ApJ, 557, 958

Galloway, D. K., Morgan, E. H., Krauss, M. I., Kaaret, P., \& Chakrabarty, D. 2007, Apj, 654, L73

Göğüs, E., Alpar, M. A., \& Gilfanov, M. 2007, ApJ, 659, 580

Heinke, C. O., et al. 2009, arXiv:0911.0444

Lamb, F. K., Boutloukos, S., Van Wassenhove, S., Chamberlain, R. T., Lo, K. H., Clare, A., Yu, W., \& Miller, M. C. 2009, ApJ, 706, 417

Markwardt, C. B., Altamirano, D., Swank, J. H., Strohmayer, T. E., Linares, M., \& Pereira, D. 2009, The Astronomer's Telegram, 2197, 1

Patruno, A., Wijnands, R., \& van der Klis, M. 2009, ApJ, 698, L60

Poutanen, J., Ibragimov, A., \& Annala, M. 2009, ApJ, 706, L129

Titarchuk, L., Cui, W., \& Wood, K. 2002, ApJ, 576, L49

Titarchuk, L., Kuznetsov, S., \& Shaposhnikov, N. 2007, ApJ, 667, 404

Torres, M. A. P., Jonker, P. G., Steeghs, D., Simon, J. D., \& Gutowski, G. 2009a, The Astronomer's Telegram, 2216, 1

Torres, M. A. P., Jonker, P. G., Steeghs, D., Damjanov, I., Caris, E., \& Glazebrook, K. 2009b, The Astronomer's Telegram, 2233, 1

Watts, A. L., Altamirano, D., Markwardt, C. B., \& ., Strohmayer, T. E. S. 2009, The Astronomer's Telegram, 2199, 1

Wijnands, R. 2008a, A Population Explosion: The Nature \& Evolution of X-ray Binaries in Diverse Environments, 1010, 382

Wijnands, R. 2008b, American Institute of Physics Conference Series, 1068, 231

Wijnands, R., \& van der Klis, M. 1998, Nature, 394, 344 